\title{
ASYMPTOTIC DEPENDENCE OF MOVING AVERAGE TYPE SELF-SIMILAR STABLE RANDOM FIELDS*
}

\author{
PIOTR S. KOKOSZKA AND MURAD S. TAQQU
}

\section{Introduction and main results}

As non-Gaussian stable stochastic processes have infinite second moments, one cannot use the covariance function to describe their dependence structure. We focus instead on the function

$$
\begin{aligned}
r(u) & =r\left(X, u ; \theta_{1}, \theta_{2}\right) \\
& =E \exp \left\{i\left[\theta_{1} X(u)+\theta_{2} X(0)\right]\right\} \\
& -E \exp \left\{i [ \theta _ { 1 } X ( u ) \} E \operatorname { e x p } \left\{i\left[\theta_{2} X(u)\right\}, u \in \mathbf{R}^{1} ; \theta_{1}, \theta_{2} \in \mathbf{R}^{1},\right.\right.
\end{aligned}
$$

which is defined for any stationary process $\left\{X(u), u \in \mathbf{R}^{1}\right\}$.

This paper investigates the asymptotic behavior, as $u \rightarrow \infty$, of $r(u)$ for a large class of self-similar stable processes obtained as 'projections' of random fields. The function $r(u)$ is the difference between the characteristic function of the vector $(X(u), X(0))$ and the product of the characteristic functions of $X(u)$ and $X(0)$; it vanishes if and only if $X(u)$ and $X(0)$ are independent. If $\{X(u)\}$ is a Gaussian process, then $r(u)$ is asymptotically proportional to the covariance, provided the latter tends to zero, as $u \rightarrow \infty$. (See Levy and Taqqu [6], Theorem 1.1.)

The present section contains definitions, statements of the main results, and some comments. The proofs are given in Section 2 .

A random field $\left\{X(t), t \in \mathbf{R}^{n}\right\}$ is called $S \alpha S$ (symmetric $\alpha$-stable) if any linear combination $\sum_{j=1}^{d} \theta_{j} X\left(t_{j}\right)$ has a symmetric stable distribution. We say that $\left\{X(t), t \in \mathbf{R}^{n}\right\}$ is self-similar with exponent $H$ if

$$
\forall c>0 \quad\left\{X(c t), t \in \mathbf{R}^{n}\right\} \stackrel{d}{=}\left\{c^{H} X(t), t \in \mathbf{R}^{n}\right\},
$$

and has stationary increments if

Received May 6, 1992.

* The first author is on leave from the Hugo Steinhaus Center, Poland. The second author was partially supported by the ONR Grant N00014-90-J-1287 at Boston University and by a grant of the United States-Israel Binational Science Foundation. 
(1.3) $\forall h \in \mathbf{R}^{n} \quad\left\{X(t+h)-X(h), t \in \mathbf{R}^{n}\right\} \stackrel{d}{=}\left\{X(t)-X(0), t \in \mathbf{R}^{n}\right\}$.

In (1.2) and (1.3) “ $\stackrel{d}{=}$ " denotes the equality of finite dimensional distributions. Any field satisfying both (1.2) and (1.3) is called $\mathrm{H}$-sssi.

A necessary and sufficient condition for the existence of a non-trivial $S \alpha S$ random field is

$$
(\boldsymbol{\alpha}, H) \in\{0<H<1,0<\alpha \leq 2\} \cup\left\{0<H \leq \frac{1}{\boldsymbol{\alpha}}, 0<\alpha \leq 2\right\}
$$

(see Takenaka [8], Proposition 1).

The Chentsov type random fields introduced by Takenaka (see Takenaka [8]) are examples of $S \alpha S \mathrm{H}$-sssi random fields with $0<H<1 / \alpha, 0<\alpha \leq 2$. Their dependence structure is described in Kokoszka and Taqqu [3]. The fields we study here have parameters $\alpha$ and $H$ in the first region, namely $H \in(0,1), \alpha \in(0,2]$.

Let $p$ be any norm on $\mathbf{R}^{n}$ and $M$ a $S \alpha S$ random measure on $\mathbf{R}^{n}$ with Lebesgue measure as its control measure, i.e. for any measurable function $f: \mathbf{R}^{n} \rightarrow \mathbf{R}^{1}$ satisfying $\int_{\mathbf{R}^{n}}|f(x)|^{\alpha} d x<\infty$,

$$
E \exp \left\{i \int_{\mathbf{R}^{n}} f(x) M(d x)\right\}=\exp \left\{-\int_{\mathbf{R}^{n}}|f(x)|^{\alpha} d x\right\}
$$

Consider the random field

$$
X(t)=\int_{\mathbf{R}^{n}}\left[p(x-t)^{H-\alpha / n}-p(x)^{H-\alpha / n}\right] M(d x), t \in \mathbf{R}^{n}
$$

The following proposition shows that the field (1.4) is well-defined.

Proposition 1.1. Let $p$ be a norm on $\mathbf{R}^{n}$. Then for any $n>1, H \in(0,1), a \in$ $(0,2]$,

$$
\forall t \in \mathbf{R}^{n} \quad \int_{\mathbf{R}^{n}}\left|p(x-t)^{H-n / \alpha}-p(x)^{H-n / \alpha}\right|^{\alpha} d x<\infty .
$$

Using the homogeneity of $p$ and the shift invariance of the Lebesgue measure one can easily check that the field (1.4) is H-sssi.

Random fields (1.4) with $p$ being the Euclidean norm were introduced in Takenaka [8]. By allowing $p$ to be any norm we obtain a much richer family of fields (1.4). We show, in Kokoszka and Taqqu [2] that there is a one-to-one correspondence between norms $p$ and the finite-dimensional distributions of fields (1.4). Thus, the set of all fields (1.4) has the cardinality of the continuum. Because 
we make no special assumptions on $p$ in this paper, our result applies to all fields of the form (1.4).

Now, for any vector $e \in \mathbf{R}^{n}$, define the stationary 'projection' process

$$
\begin{aligned}
X_{e}(u) & =X((u+1) e)-X(u e) \\
& =\int_{\mathbf{R}^{n}}\left[p(x-(u+1) e)^{H-n / \alpha}-p(x-u e)^{H-n / \alpha}\right] M(d x) .
\end{aligned}
$$

Our main result shows that the function $r(u)$ for the process $X_{e}$ is asymptotically proportional, as $u \rightarrow \infty$, to the power function $u^{\alpha H-\alpha}$.

Theorem 1.1. For the process $\left\{X_{e}(u), u \in \mathbf{R}^{1}\right\}$ defined in (1.6) and any $n>1$,

$$
\lim _{u \rightarrow \infty} \frac{r\left(X_{e}, u ; \theta_{1}, \theta_{2}\right)}{u^{\alpha H-\alpha}}=-e^{-c\left(\theta_{1}, \theta_{2}\right)} \int_{\mathbf{R}^{n}} \tilde{\phi}\left(y ; \theta_{1}, \theta_{2}\right) d y,
$$

where

$$
\begin{gathered}
c\left(\theta_{1}, \theta_{2}\right)=\left(\left|\theta_{1}\right|^{\alpha}+\left|\theta_{2}\right|^{\alpha}\right) \int_{\mathbf{R}^{n}}\left|p(x-e)^{H-n / \alpha}-p(x)^{H-n / \alpha}\right|^{\alpha} d x ; \\
\tilde{\phi}\left(y ; \theta_{1}, \theta_{2}\right)=\left|\theta_{1} \tilde{f}(y)+\theta_{2} \tilde{g}(y)\right|^{\alpha}-\left|\theta_{1} \tilde{f}(y)\right|^{\alpha}-\left|\theta_{2} \tilde{g}(y)\right|^{\alpha} ; \\
\tilde{g}(y)=\left(H-\frac{n}{\alpha}\right) p_{-e}^{\prime}(y) p(y)^{H-n / \alpha-1}, \tilde{f}(y)=\tilde{g}(y-e),
\end{gathered}
$$

and where

$$
p_{-e}^{\prime}(y)=\lim _{\substack{s \rightarrow 0 \\ s>0}} \frac{p(y-s e)-p(y)}{s}
$$

is the directional derivative of $p$.

Theorem 1.1 is proven in Section 2 .

Remarks:

- The limit in (1.8) exists because since $p$ is a norm, the function $\phi(s)=p(y-s e)$, $s \in \mathbf{R}^{1}$ is convex and hence has one-sided derivatives at every $s \in \mathbf{R}^{1}$.

- For a number of norms $p$ one can compute the directional derivative $p_{-e}^{\prime}(y)$. In what follows we assume $y \neq 0$, as the definition of $\tilde{g}$ does not admit $y=0$.

If $p$ is the Euclidean norm $\|\cdot\|$ then $p_{-e}^{\prime}(y)=-\|y\|^{-1}(y, e)$, where $(\cdot, \cdot)$ denotes the Euclidean scalar product. More generally, if $p$ is of the form 


$$
p(y)=\left(\int_{S_{n}}|(y, s)|^{r} \sigma(d s)\right)^{1 / r}, r \geq 1,
$$

where $S_{n}=\left\{s \in \mathbf{R}^{n}:\|s\|=1\right\}$ and $\sigma$ is a positive finite Borel measure on $S_{n}$, then

$$
p_{-e}^{\prime}(y)= \begin{cases}\int_{\{(y, s)=0\}}|(e, s)| \sigma(d s)-\int_{\{(y, s) \neq 0\}}(e, s) \operatorname{sign}(y, s) \sigma(d s), & \text { if } r=1 \\ p^{1-r}(y) \int_{S_{n}}(e, s)|(y, s)|^{r-1} \operatorname{sign}(y, s) \sigma(d s), & \text { if } r>1 .\end{cases}
$$

The norms $p$ admitting representation (1.9) are characterized in Kokoszka and Taqqu [2]. They generate random fields (1.4) which can be considered extensions to the parameter space $\mathbf{R}^{n}$ of the fractional Lévy motion (see Kokoszka and Taqqu [2] for the details).

- Theorem 1.1 implies that $r(u)$ tends to zero, as $u \rightarrow \infty$, a fact that can also be deduced from Lemma 6.1 of Kokoszka and Taqqu [2]. That lemma states that if

$$
X(t)=\int_{\mathbf{R}^{n}} k(t-x) M(d x), t \in \mathbf{R}^{n}
$$

with the function $k$ satisfying $\int_{\mathbf{R}^{n}}|k(x)|^{\alpha} d x<\infty$, then

$$
E \exp i\left[\theta_{1} X(t)+\theta_{2} X(0)\right]-E \exp i \theta_{1} X(t) E \exp i \theta_{2} X(0) \rightarrow 0,
$$

as $\|t\| \rightarrow 0$.

- If $\alpha=2$, the rate of decay of $r(u)$ is $u^{2 H-2}$. This type of behavior in the Gaussian case $\alpha=2$ is well known. One can derive it using the following argument: The process $X_{e}$ is the one-step-increment process of an H-sssi zero mean Gaussian process

$$
\xi(u)=\int_{\mathbf{R}^{n}}\left[p(x-u e)^{H-n / 2}-p(x)^{H-n / 2}\right] M(d x)
$$

which is necessarily proportional to the fractional Brownian motion $B_{H}$ whose covariances are

$$
E\left[B_{H}\left(u_{1}\right) B_{H}\left(u_{2}\right)\right]=\frac{1}{2}\left\{\left|u_{1}\right|^{2 H}+\left|u_{2}\right|^{2 H}-\left|u_{1}-u_{2}\right|^{2 H}\right\}
$$

Therefore, the process $X_{e}$ has the covariance function 


$$
E\left[X_{e}(u) X_{e}(0)\right]=\frac{c}{2}\left\{|u+1|^{2 H}-2|u|^{2 H}+|u-1|^{2 H}\right\},
$$

which behaves like $c H(2 H-1) u^{2 H-2}$, as $u \rightarrow \infty$. As mentioned above, for any Gaussian process, $r(u)$ is proportional to the covariance function.

- Our result is valid for $n>1$. If $n=1$, then $p(x)=|x| p(1)$, so $X_{e}$ is proportional to the one-step-increment process of the well-balanced linear fractional Lévy motion

$$
L(u)=\int_{-\infty}^{\infty}\left[|x-u|^{H-1 / \alpha}-|x|^{H-1 / \alpha}\right] M(d x) .
$$

For the process $L, r(u)$ is asymptotic to

$$
\begin{aligned}
& u^{\alpha H-\alpha}, \text { if either } 0<\alpha \leq 1,0<H<1 \text {, or } \\
& \quad 1<\alpha \leqslant 2,1-[\alpha(\alpha-1)]^{-1}<H<1 \text { and } H \neq 1 / \alpha ; \\
& u^{H-\alpha^{-1}-1} \text {, if } 1<\alpha<2 \text { and } 0<H<1-[\alpha(\alpha-1)]^{-1}
\end{aligned}
$$

(see Astrauskas, Levy and Taqqu [1]).

Such a "phase transition" of the function $r(u)$ appears only in the onedimensional case $n=1$. Notice that the exponent $H-n / \alpha$ is negative for any $H \in(0,1)$ and $\alpha \in(0,2]$ when $n>1$, whereas it is positive when $n=1$ and $\alpha H>1$.

- As mentioned above, in many cases, different norms $p$ generate fields (1.4) with different finite dimensional distributions. Although Theorem 1.1 shows that the two-dimensional distributions of processes (1.6) are asymptotically proportional, they need not be proportional. Consider two processes (1.6) with parameters $\left(n_{i}, p_{i}, e_{j}\right), i=1,2$. We list below three typical cases in which

$$
\forall c \in \mathbf{R}^{1} \quad\left(X_{e_{1}}(0), X_{e_{1}}(1)\right) \stackrel{d}{\neq} c\left(X_{e_{2}}(0), X_{e_{2}}(1)\right) .
$$

1. $n_{1}=n_{2}, p_{1}=p_{2}, e_{1} \neq e_{2}$ (see Kokoszka and Taqqu [2], Example 3.2).

2. $n_{1}=n_{2}, p_{1} \neq p_{2}, e_{1}=e_{2}$ (see Kokoszka and Taqqu [2], Example 3.3).

3. $n_{1} \neq n_{2}$. In this case one cannot, of course, set $p_{1}=p_{2}, e_{1}=e_{2}$, but one can take $p_{1}$ and $p_{2}$ to be equal to the Euclidean norms in $\mathbf{R}^{n_{1}}$ and $\mathbf{R}^{n_{2}}$, respectively, and choose $e_{1}$ and $e_{2}$ so that all their coordinates are equal to 1 . 
(See Samorodnitsky and Taqqu [7], Thm. 3.1.)

- The function $r(u)$ can be used to distinguish various $S \alpha S$ H-sssi fields and processes. While all symmetric Gaussian H-sssi processes are proportional to the fractional Brownian motion $B_{H}$ defined by $(1.10)$, there are many different non-Gaussian stable H-sssi processes, and it is often not easy to show that two such processes are not proportional. One way to show it is by using $r(u)$. For example, Theorem 1.1 shows no field (1.4) with $\alpha<2$ is a Chentsov random field in the sense of Takenaka [8]. Indeed, if $\left\{Y(t), t \in \mathbf{R}^{n}\right\}$ denotes the latter field, then, for any $e \in \mathbf{R}^{n}$, the function $r(u)$ for the process $Y((u+1) e)-Y(u e), u \in \mathbf{R}^{1}$, behaves asymptotically like $u^{\alpha H-2}$ (see Kokoszka and Taqqu [3], Theorem 2.1). Other applications of this type are presented in Kokoszka and Taqqu [2] and Kokoszka and Taqqu [3].

- The asymptotic form of $r(u)$ for ARMA time series with $S \alpha S$ noises can be found in Kokoszka and Taqqu [4], where we also suggest an interpretation of the function $r(u)$.

\section{The proofs}

Throughout this section we shall use the following notation: $\|\cdot\|$ denotes the Euclidean norm in $\mathbf{R}^{n} ; S_{n}$ is the unit sphere in $\mathbf{R}^{n}$, i.e. $S_{n}=\left\{s \in \mathbf{R}^{n}:\|s\|=1\right\}$; $d s$ stands for the spherical Lebesgue measure on $S_{n}$. Thus, for any positive measurable function $f: \mathbf{R}^{n} \rightarrow \mathbf{R}_{+}$

$$
\int_{\mathbf{R}^{n}} f(x) d x=\int_{0}^{\infty}\left[\int_{S_{n}} f(r s) d s\right] r^{n-1} d r .
$$

For the sake of brevity, we set

$$
\gamma:=\frac{n}{\alpha}-H
$$

Notice that for any $\alpha \in(0,2], H \in(0,1), \gamma>0$, provided $n>1$. In the sequel we always assume $n>1$.

Here are a number of simple facts that will be frequently used:

Lemma 2.1. For any $\alpha \in(0,2]$ and $H \in(0,1)$,

(a) $\alpha \gamma<n$;

(b) $\alpha(\gamma+1)>n$. 
If, in addition $\alpha>1$, then

(c) $0<\gamma(\alpha-1)<\frac{n}{2}$.

Also, for any $\gamma>0$ :

$$
\alpha^{-\gamma}-b^{-\gamma}=\gamma(b-a) \xi^{-(\gamma+1)}, \quad a, b>0,
$$

where $\min (a, b)<\xi<\max (a, b)$.

Throughout the paper $p$ denotes a norm on $\mathbf{R}^{n}$. We often use the scaling property $p(k x)=k p(x), k>0$ and the fact that $p$ is equivalent to the Euclidean norm on $\mathbf{R}^{n}$ i.e. there are positive constants $c_{0}$ and $c_{1}$ such that

$$
c_{0}\|x\| \leq p(x) \leq c_{1}\|x\|, x \in \mathbf{R}^{n}
$$

(see, for example, Kreyzig [5]).

We start with the proof of Proposition 1.1, which is followed by that of Theorem 1.1.

Proof of Proposition 1.1. We must show

$$
\forall t \in \mathbf{R}^{n} \quad \int_{\mathbf{R}^{n}}\left|p(x-t)^{-r}-p(x)^{-r}\right|^{\alpha} d x<\infty .
$$

One can, of course, assume $t \neq 0$. Since $\gamma>0$, we have to check the convergence of the integral in some neighborhoods of points $0, t$ and $\infty$. It is more convenient to set $x=r s, r>0, s \in S_{n}$, and work with the spherical coordinates $(r, s)$.

For small $r$,

$$
p(r s-t)^{-r}-p(r s)^{-r}=r^{-r} p(s)^{-r}\left[\left(\frac{r p(s)}{p(r s-t)}\right)^{r}-1\right] .
$$

Since by the triangle inequality $\left|\frac{r p(s)}{p(r s-t)}\right| \leq \frac{c_{1} r}{p(t)-c_{0} r} \rightarrow 0$, as $r \rightarrow 0$, we have $\left|\left(\frac{r p(s)}{p(r s-t)}\right)^{r}-1\right|<1$ for $r<r_{0}$ and any $S \in S_{n}$, and so

$$
\begin{gathered}
\int_{0}^{r_{0}}\left(\int_{S_{n}}\left|p(r s-t)^{-r}-p(r s)^{-r}\right|^{\alpha} d s\right) r^{n-1} d r \\
<c_{0}^{-r \alpha} \int_{0}^{r_{0}} r^{-r \alpha+n-1} d r<\infty
\end{gathered}
$$

by Lemma 2.1 (a).

Thus, the integral in (2.5) is finite in a neighborhood of zero. By the shift 
invariance of Lebesgue measure it is also finite in a neighborhood of $t$.

For large $\boldsymbol{r}$, we use (2.3) and write

$$
p(r s-t)^{-r}-p(r s)^{-r}=\gamma[p(r s)-p(r s-t)] \xi^{-(r+1)},
$$

for some $\xi>c_{0} r-p(t)$. Thus, there are constants $k$ and $r_{1}$ depending on $t$ such that for $r>r_{1}, \xi^{-(\gamma+1)}<K r^{-(\gamma+1)}$. Since $|p(r s)-p(r s-t)|<p(t)$, (2.6) yields

$$
\left|p(r s-t)^{-r}-p(r s)^{-r}\right|^{\alpha}<(\gamma p(t) K)^{\alpha} r^{-\alpha(r+1)} .
$$

Therefore, by Lemma 2.1(b), the integral in (2.5) is finite in a neighborhood of infinity.

Although in the sequel we invoke neither formula (2.1) nor inequalities (2.4), they are implicitly used in most arguments dealing with the convergence of integrals.

The remainder of the paper is devoted to the proof of Theorem 1.1.

Proof of Theorem 1.1. Notice that the function $r(u)=r\left(X_{e}, u ; \theta_{1}, \theta_{2}\right)$ defined by (1.1) can be factored as follows:

$$
r(u)=e^{-c\left(\theta_{1}, \theta_{2}\right)}\left(e^{-I(u)}-1\right),
$$

where

$$
\begin{aligned}
c\left(\theta_{1}, \theta_{2}\right) & =-\left[\ln E \exp i \theta_{1} X_{e}(u)+\ln E \exp i \theta_{2} X_{e}(0)\right] \\
& =\left(\left|\theta_{1}\right|^{\alpha}+\left|\theta_{2}\right|^{\alpha}\right) \int_{\mathbf{R}^{n}}\left|p(x-e)^{-r}-p(x)^{-\gamma}\right|^{\alpha} d x,
\end{aligned}
$$

and

$$
\begin{aligned}
I(u)=I\left(X_{e}, u ; \theta_{1}, \theta_{2}\right) & =-\ln E \exp i\left[\theta_{1} X_{e}(u)+\theta_{2} X_{e}(0)\right] \\
& +\ln E \exp i \theta_{1} X_{e}(u)+\ln E \exp i \theta_{2} X_{e}(0) .
\end{aligned}
$$

Setting $a(u, x)=p(x-(u+1) e)^{-\gamma}-p(x-u e)^{-\gamma}$, we have

$$
X_{e}(u)=\int_{\mathbf{R}^{n}} a(u, x) M(d x)
$$

and so

$$
I(u)=\int_{\mathbf{R}^{n}}\left[\left|\theta_{1} a(u, x)+\theta_{2} a(0, x)\right|^{\alpha}-\left|\theta_{1} a(u, x)\right|^{\alpha}-\left|\theta_{2} a(0, x)\right|^{\alpha}\right] d x .
$$

After setting $y=u^{-1} x$ and taking into account (2.2), (2.8) becomes 


$$
I(u)=u^{\alpha H-\alpha} \int_{\mathbf{R}^{n}} \phi(u, y) d y,
$$

where

$$
\begin{aligned}
\phi(u, y) & =\phi\left(u, y ; \theta_{1}, \theta_{2}\right) \\
& =\left|\theta_{1} f(u, y)+\theta_{2} g(u, y)\right|^{\alpha}-\left|\theta_{1} f(u, y)\right|^{\alpha}-\left|\theta_{2} g(u, y)\right|^{\alpha},
\end{aligned}
$$

and

$$
g(u, y)=u\left[p\left(y-u^{-1} e\right)^{-r}-p(y)^{-r}\right],
$$

$$
f(u, y)=u\left[p\left(y-\left(1+u^{-1}\right) e\right)^{-r}-p(y-e)^{-\gamma}\right]=g(u, y-e) .
$$

Now suppose that

$$
\lim _{u \rightarrow \infty} \int_{\mathbf{R}^{n}} \phi(u, y) d y=\int_{\mathbf{R}^{n}} \tilde{\phi}(y) d y
$$

where $\tilde{\phi}(y)=\tilde{\phi}\left(y ; \theta_{1}, \theta_{2}\right)$ is defined in the statement of Theorem 1.1. If (2.13) holds, then, by (2.9), $I(u) \rightarrow 0$, and so Theorem 1.1 follows from (2.7). Thus, to establish Theorem 1.1 it is sufficient to prove (2.13). We shall do it in a number of lemmas.

Lemma 2.2. If $\phi$ and $\tilde{\phi}$ are as defined above, then

$$
\forall y \in \mathbf{R}^{n} \backslash\{0, e\} \quad \lim _{u \rightarrow \infty} \phi(u, y)=\tilde{\phi}(y) .
$$

Proof. In view of (2.11) and (2.12), it suffices to check that

$$
\forall y \neq 0 \quad \lim _{u \rightarrow \infty} g(u, y)=\tilde{g}(y)
$$

Recall that

$$
\tilde{g}(y)=-\gamma p_{-e}^{\prime}(y) p(y)^{-(\gamma+1)},
$$

where

$$
p_{-e}^{\prime}(y)=\lim _{s \rightarrow 0, s>0} \frac{p(y-s e)-p(y)}{s}
$$

Now, using (2.3), we have

$$
g(u, y)=\gamma u\left[p(y)-p\left(y-u^{-1} e\right)\right] \xi_{u}^{-(\gamma+1)}(y),
$$


where $\xi_{u}(y)$ is a point lying between $p(y)$ and $p\left(y-\frac{1}{u} e\right)$ and hence $\lim _{u \rightarrow \infty} g(u, y)$ $=-\gamma p_{-e}^{\prime} \lim _{u \rightarrow \infty} \xi_{u}(y)^{-(\gamma+1)}$. Since $p$ is continuous, $\lim _{u \rightarrow \infty} \xi_{u}(y)=p(y)$, and so (2.14) follows.

We shall use the following inequalities:

$$
\begin{gathered}
\left|u\left(p(y)-p\left(y-u^{-1} e\right)\right)\right| \leq p(e) ; \\
\left|p_{-e}^{\prime}(y)\right| \leq p(e) .
\end{gathered}
$$

Inequality (2.17) follows from the triangle inequality and (2.18) from (2.17). We shall also extensively use the following inequalities which hold for any real numbers $r$ and $s$

$$
\| r+\left.s\right|^{\alpha}-|r|^{\alpha}-|s|^{\alpha} \mid \leq \begin{cases}2|r|^{\alpha}, & \text { for } \alpha \in(0,1] \\ \alpha|r||s|^{\alpha-1}+(a+1)|r|^{\alpha} & \text { for } \alpha \in(1,2]\end{cases}
$$

To prove (2.13) we shall show that for each $\lambda \in(0,1)$,

$$
\lim _{u \rightarrow \infty} \int_{\{p(y-e)>\lambda p(e)\}}[\phi(u, y)-\tilde{\phi}(y)] d y=0
$$

and

$$
\lim _{u \rightarrow \infty} \int_{\{p(y-e)<\lambda p(e)\}}[\phi(u, y)-\tilde{\phi}(y)] d y=0 .
$$

We shall first focus on the proof of (2.20) which consists of a number of lemmas.

Lemma 2.3. For each $\lambda \in(0,1)$,

$$
\int_{\{p(y-e)>\lambda p(e)\}}\left(\sup _{u>2 \lambda^{-1}}|f(u, y)|^{\alpha}\right) d y<\infty .
$$

Proof. It is sufficient to find constants $K_{1}$ and $K_{2}$ such that for $u>2 \lambda^{-1}$ and $p(y-e)>\lambda p(e)$,

$$
|f(u, y)| \leq K_{1}
$$

and

$$
|f(u, y)| \leq K_{2} p(y)^{-(\gamma+1)} \text {, provided } p(y)>2 p(e) .
$$

The lemma then follows because by Lemma 2.1(b), $-(\gamma+1) \alpha+n-1<-1$. 
Using (2.3) and (2.17), we get

$$
|f(u, y)| \leq \gamma p(e)\left(\min \left[p(y-e), p\left(y-\left(1+u^{-1}\right) e\right)\right]\right)^{-(\gamma+1)}
$$

Since $p(y-e)>\lambda p(e)$ and, as $u>2 \lambda^{-1}, p\left(y-\left(1+\frac{1}{u}\right) e\right) \geq p(y-e)-$ $\frac{1}{u} p(e)>\lambda p(e)-\frac{1}{u} p(e)>\frac{\lambda}{2} p(e),(2.24)$ shows that (2.22) holds with $K_{1}=$ $\gamma 2^{r+1} \lambda^{-(\gamma+1)} p(e)^{-r}$. If $p(y)>2 p(e)$, then $p(y-e)>\frac{1}{2} p(y)$ and, for $u>2 \lambda^{-1}$, $p\left(y-\left(1+\frac{1}{u}\right) e\right)>\left(\frac{1}{2}-\frac{\lambda}{4}\right) p(y)>\frac{1}{4} p(y)$. Again (2.24) yields (2.23).

Relation (2.20) for $\alpha \in(0,1]$ follows from Lemma 2.3, inequality (2.19) with $r=f(u, y)$, Lemma 2.2 and the Dominated Convergence Theorem. The case of $\alpha \in(1,2]$ is more difficult because one must apply inequalities (2.19) with $s=g(u, y)$, a function which behaves badly around $y=0$, as $u \rightarrow \infty$. To prove (2.20) for $\alpha>1$ we shall assume $u$ large enough, choose $\delta$ and $\varepsilon$ so that $0<2 u^{-1}$ $<\delta<\varepsilon \leq 2$ and integrate separately over the regions $\left\{p(y)<2 u^{-1} p(e)\right\}$, $\left\{2 u^{-1} p(e)<p(y)<\delta p(e)\right\},\{\delta p(e)<p(y)<\varepsilon p(e)\},\{p(y)>2 p(e)\}$, while excluding the ball $\{p(y-e)<\lambda p(e)\}$ with radius $\lambda p(e)$ and center $e$.

LEMMA 2.4.

$$
R(u)=\int_{\left\{p(y)<\frac{2}{u} p(e)\right\}}[|\phi(u, y)|+|\tilde{\phi}(y)|] d y
$$

tends to 0 , as $u \rightarrow \infty$.

Proof. Choose $u_{0}>2 \lambda^{-1}$ so large that for $u>u_{0}$

$$
\left\{p(y)<2 u^{-1} p(e)\right\} \subset\{p(y-e)>\lambda p(e)\} \cap\{p(y) \leq 2 p(e)\} .
$$

Then, by (2.22), the functions $|f(u, y)|, u>u_{0}$ are bounded on $\{p(y)<$ $\left.\frac{2}{u} p(e)\right\}$ by a constant which does not depend on $u$. Since the measure of the set $\left\{p(y)<\frac{2}{u} p(e)\right\}$ tends to zero, we have

$$
\lim _{u \rightarrow \infty} \int_{\left\{p(y)<\frac{2}{u} p(e)\right\}}\left[|f(u, y)|^{\alpha}+|\tilde{f}(y)|^{\alpha}\right] d y=0 .
$$

We shall now show that 


$$
\lim _{u \rightarrow \infty} \int_{\left\{p(y)<\frac{2}{u} p(e)\right\}}|g(u, y)|^{\alpha-1} d y=0
$$

and

$$
\lim _{u \rightarrow \infty} \int_{\left\{p(y)<\frac{2}{u} p(e)\right\}}|\tilde{g}(y)|^{\alpha-1} d y=0 .
$$

Notice that once (2.27) and (2.28) have been proven, the lemma will follow by applying inequalities (2.19) first with $r=|f(u, y)|$ and $s=|g(u, y)|$, then with $r=|\tilde{f}(y)|$ and $s=|\tilde{g}(y)|$.

To prove (2.27) note that

$$
|g(u, y)| \leq u\left[p(y)^{-r}+p\left(y-u^{-1} e\right)^{-r}\right]
$$

implies for $\alpha \in(1,2]$,

$$
\begin{gathered}
\int_{\left\{p(y)<\frac{2}{u} p(e)\right\}}|g(u, y)|^{\alpha-1} d y \\
\leq u^{\alpha-1}\left[\int_{\left\{p(y)<\frac{2}{u} p(e)\right\}} p(y)^{-\gamma(\alpha-1)} d y+\int_{\left\{p(y)<\frac{2}{u} p(e)\right\}} p\left(y-u^{-1} e\right)^{-r(\alpha-1)} d y\right] \\
=u^{\alpha-1-n+\gamma(\alpha-1)}\left[\int_{\{p(x)<2 p(e)\}} p(x)^{-\gamma(\alpha-1)} d x+\int_{\{p(x)<2 p(e)\}} p(x-e)^{-\gamma(\alpha-1)} d x\right] .
\end{gathered}
$$

As, by Lemma 2.1(c), $-\gamma(\alpha-1)+n-1>-1$ and $\alpha-1-n+\gamma(\alpha-1)$ $<0$, (2.27) follows. To show (2.28), it is enough to check that the function $|\tilde{g}(y)|^{a-1}$ is integrable in a neighborhood of zero. This, however, follows immediately from (2.15), (2.18) and Lemma 2.1(c).

Lemma 2.5. Fix $0<\delta<2$ and define

$$
D_{\delta}(u)=\int_{\Omega(u, \delta)}|\phi(u, y)-\tilde{\phi}(y)| d y, u>2 \delta^{-1},
$$

where

(2.30) $\Omega(u, \delta)=\{p(y-e)>\lambda p(e)\} \cap\left\{2 u^{-1} p(e)<p(y)<\delta p(e)\right\}, u>2 \delta^{-1}$.

Then,

$$
\lim _{\delta \rightarrow 0}\left(\sup _{u>2 \max \left(\lambda^{-1}, \delta^{-1}\right)} D_{\delta}(u)\right)=0 .
$$


Proof. In view of inequalities (2.19) it suffices to check that

$$
\begin{gathered}
\lim _{\delta \rightarrow 0}\left(\sup _{u>2 \max \left(\lambda^{-1}, \delta^{-1}\right)} \int_{\Omega(u, \delta)}|f(u, y)|^{\alpha} d y\right)=0 ; \\
\lim _{\delta \rightarrow 0}\left(\sup _{u>2 \max \left(\lambda^{-1}, \delta^{-1}\right)} \int_{\Omega(u, \delta)}|\tilde{f}(y)|^{\alpha} d y\right)=0 ; \\
\lim _{\delta \rightarrow 0}\left(\sup _{u>2 \max \left(\lambda^{-1}, \delta^{-1}\right)} \int_{\Omega(u, \delta)}|g(u, y)|^{\alpha-1}|f(u, y)| d y\right)=0 ; \\
\lim _{\delta \rightarrow 0}\left(\sup _{u>2 \max \left(\lambda^{-1}, \delta^{-1}\right)} \int_{\Omega(u, \delta)}|\tilde{g}(y)|^{\alpha-1}|\tilde{f}(y)| d y\right)=0 .
\end{gathered}
$$

As the measure of the sets $\Omega(u, \delta)$ tends to zero, as $\delta \rightarrow 0,(2.31)$ and (2.32) follow from (2.22). In the proof of Lemma 2.4 we have shown that $|\tilde{g}(y)|^{a-1}$ is integrable in a neighborhood of zero, so (2.34) also holds. To prove (2.33), notice that $p(y)>\frac{2}{u} p(e)$ implies $p\left(y-\frac{1}{u} e\right)>\frac{1}{2} p(y)$, so by (2.3) and (2.17), for each $y \in \Omega(u, \delta)$,

$$
\sup _{u>2 \delta^{-1}}|g(u, y)| \leq \gamma p(e) 2^{\gamma+1} p(y)^{-(r+1)},
$$

and Lemma 2.1(c) again completes the proof.

Lemma 2.6. Fix $0<\delta<\varepsilon \leq 2$ and define

$$
H_{\delta, \varepsilon}(u)=\int_{\Delta(\delta, \varepsilon)}|\phi(u, y)-\tilde{\phi}(y)| d y
$$

where

$$
\Delta(\delta, \varepsilon)=\{p(y-e)>\lambda p(e)\} \cap\{\delta p(e)<p(y)<\varepsilon p(e)\}
$$

Then,

$$
\lim _{u \rightarrow \infty} H_{\delta, \varepsilon}(u)=0
$$

Proof. By (2.3),

$$
g(u, y)=r u\left[p(y)-p\left(y-u^{-1} e\right)\right] \xi_{u}^{-(\gamma+1)}(y),
$$

where $\xi_{u}(y)$ is a point lying between $p(y)$ and $p\left(y-\frac{1}{u} e\right)$. If $y \in \Delta(\delta, \varepsilon)$, then 
clearly, $p(y)>\frac{1}{2} \delta p(e)$, and if, in addition, $u>2 \delta^{-1}$, then $p\left(y-\frac{1}{u} e\right)>p(y)-$ $\frac{1}{u} p(e)>\frac{1}{2} \delta p(e)$. Thus, $\xi_{u}(y)>\frac{1}{2} \delta p(e)$, and so, by (2.17),

$$
|g(u, y)| \leq 2^{\gamma+1} \gamma \delta^{-(\gamma+1)} p(e)^{-\gamma} .
$$

To complete the proof combine inequalities (2.38) and (2.22) with inequalities (2.19) applied to $r=|f(u, y)|$ and $s=|g(u, y)|$ and apply the Dominated Convergence Theorem.

Corollary 2.1. For $\lambda \in(0,1)$ define

$$
A(\lambda)=\{p(y-e)>\lambda p(e)\} \cap\{p(y)<2 p(e)\} .
$$

Then, $\lim _{u \rightarrow 0} \int_{A(\lambda)}[\phi(u, y)-\tilde{\phi}(y)] d y=0$.

Proof. For any $\delta \in(0,2)$ and sufficiently large $u$

$$
A(\lambda)=\left\{p(y)<2 u^{-1} p(e)\right\} \cup \Omega(\delta) \cup \Delta(\delta, 2),
$$

so

$$
\left|\int_{A(\lambda)}[\phi(u, y)-\tilde{\phi}(y)] d y\right| \leq R(u)+D_{\delta}(u)+H_{\delta, 2}(u)
$$

By Lemmas 2.4 and 2.6,

$$
\limsup _{u \rightarrow \infty}\left|\int_{A(\lambda)}[\phi(u, y)-\tilde{\phi}(y)] d y\right| \leq \sup _{u>2 \lambda^{-1}} D_{\delta}(u) .
$$

To complete the proof, let $\delta \rightarrow 0$ and use Lemma 2.5.

Lemma 2.7. One has

$$
\lim _{u \rightarrow \infty} \int_{\{p(y)>2 p(e)\}}[\phi(u, y)-\tilde{\phi}(y)] d y=0 .
$$

Proof. We shall first show that for $\alpha \in(1,2]$,

$$
\int_{\{p(y)>2 p(e)\}}\left\{\sup _{u>2}|f(u, y)||g(u, y)|^{\alpha-1}\right) d y<\infty .
$$

Using (2.3) and inequality (2.17) we get 


$$
\begin{aligned}
& |f(u, y)| \leq \gamma p(e) \xi_{u}^{-(\gamma+1)}(y) ; \\
& |g(u, y)| \leq \gamma p(e) \eta_{u}^{-(\gamma+1)}(y),
\end{aligned}
$$

where $\xi_{u}(y)$ lies between $p(y-e)$ and $p\left(y-\left(1+\frac{1}{u}\right) e\right)$, and $\eta_{u}$ lies between $p(y)$ and $p\left(y-\frac{1}{u} e\right)$. Since $p(y)>2 p(e), p(y-e)>\frac{1}{2} p(y)$ and for $u>2$, $p\left(y-\left(1+\frac{1}{u}\right) e\right) \geq p(y)-\left(1+\frac{1}{u}\right) p(e)>p(y)-\frac{3}{2} p(e)>\frac{1}{4} p(y)$, so $\xi_{u}(y)>$ $\frac{1}{4} p(y)$. By a similar argument $\eta_{u}(y)>\frac{3}{4} p(y)>\frac{1}{4} p(e)$. Thus,

$$
\begin{aligned}
& |f(u, y)| \leq \gamma p(e) 4^{\gamma+1} p(y)^{-(\gamma+1)} ; \\
& |g(u, y)| \leq \gamma p(e) 4^{\gamma+1} p(y)^{-(\gamma+1)},
\end{aligned}
$$

whenever $u>2$. Since $-(\gamma+1) \alpha+n-1<1$ by Lemma 2.1(b), (2.39) follows. By the same argument,

$$
\int_{\{p(y)>2 p(e)\}}\left(\sup _{u>2}|f(u, y)|^{\alpha}\right) d y<\infty
$$

so the lemma follows from inequalities (2.19) and the Dominated Convergence Theorem.

Notice that Lemma 2.7 and Corollary 2.1 yield relation (2.20). Thus to prove (2.13) it remains to establish (2.21). To prove $(2.21)$ (for any $\lambda \in(0,1))$ use relations $f(u, y)=g(u, y-e)$ and $\tilde{f}(y)=\tilde{g}(y-e)$ to verify statements analogous to Lemmas $2.4,2.5$ and 2.6 with the corresponding sets centered at vector $e$, namely:

$$
\begin{gathered}
\lim _{u \rightarrow \infty} \int_{\left\{p(y-e)<2 u^{-1} p(e)\right\}}[|\phi(u, y)|+|\tilde{\phi}(y)|] d y=0 ; \\
\lim _{\delta \rightarrow 0}\left(\sup _{u>2 \max \left(\delta^{-1},(1-\lambda)^{-1}\right)} \int_{\left\{2 u^{-1}<p(y-e)<\delta p(e)\right\}}|\phi(u, y)-\tilde{\phi}(y)| d y\right)=0,0<\delta<\lambda ; \\
\lim _{u \rightarrow \infty} \int_{\{\delta p(e)<p(y-e)<\lambda p(e)\}}[\phi(u, y)-\tilde{\phi}(y) \mid d y=0 .
\end{gathered}
$$

Having proven Relation (2.13), we have completed the proof of Theorem 1.1. 


\section{REFERENCES}

[1] A. Astrauskas, J. B. Levy, and M. S. Taqqu, The asymptotic dependence structure of the linear fractional Lévy motion, Lietuvos Matematikos Rinkinys (Lithuanian Mathematical Journal), 31 (1) (1991), 1-28.

[2] P. S. Kokoszka and M. S. Taqqu, New classes of self-similar symmetric stable random fields, to appear in J. Theoretical Prob., 1993.

[3] - Asymptotic dependence of statble self-similar processes of Chentsov type, In M. G. Hahn R. M. Dudley and J. Kuelbs, editors, Probability in Banach Spaces, 8: Proceedings of the Eighth International Conference, pages 152-165, Boston, 1992. Birkhäuser.

[4] - Infinite variance stable ARMA processes, to appear in Journal for Time Series Analysis, 1993.

[5] E. Kreyzig, Introductory Functional Analysis with Applications, Wiley \& Sons, 1989.

[6] J. Levy and M. S. Taqqu, A characterization of the asymptotic behavior of stationary stable processes, In G. Samorodnitsky S. Cambanis and M. S. Taqqu, editors, Stable Processes and Related Topics, volume 25 of Progress in Probability, pages 181-198, Boston, 1991. Birkhäuser.

[ 7 ] G. Samorodnitsky and M. S. Taqqu, $(1 / \alpha)$-self-similar processes with stationary increments, J. Multivariate Anal. 35 (1990), 308-313.

[8] S. Takenaka, Integral-geometric construction of self-similar stable processes, Nagoya Math. J., 123 (1991), 1-12.

Piotr S. Kokoszka

Boston University

Department of Mathematics

111 Cummington Street

Boston, MA 02215-2411, USA

Email:piotr@math.bu.edu

Murad S. Taqqu

Boston University

Department of Mathematics

111 Cummington Street

Boston, MA 02215-2411, USA

Email:murad@math.bu.edu 